\title{
Isospin effects on sub-threshold kaon production at intermediate energies
}

\author{
G. Ferini ${ }^{1}$, T. Gaitanos ${ }^{2}$, M. Colonna ${ }^{1}$, M. Di Toro ${ }^{1}$, H.H. Wolter ${ }^{2}$ \\ 1 Università di Catania and INFN, \\ Lab. Nazionali del Sud, 95123 Catania, Italy \\ 2 Dept. für Physik, Universität München, \\ D-85748 Garching, Germany \\ E-mail: ditoro@lns.infn.it
}

\begin{abstract}
We show that in collisions with neutron rich heavy ions at energies around the production threshold $K^{0}$ and $K^{+}$yields might probe the isospin dependent part of the nuclear Equation of State $(E o S)$ at high baryon densities. In particular we suggest the $K^{0} / K^{+}$ratio as a promising observable. Results obtained in a fully covariant relativistic transport approach are presented for central $A u+A u$ collisions in the beam energy range $0.8-1.8 \mathrm{AGeV}$. The focus is put on the EoS influence which goes beyond the collision - cascade picture. The isovector part of the in-medium interaction affects the kaon multiplicities via two mechanisms: i) a symmetry potential effect, i.e. a larger neutron repulsion in n-rich systems (isospin fractionation); ii) a threshold effect, due to the change in the self-energies of the particles involved in inelastic processes. Genuine relativistic contributions are revealed, that could allow to directly "measure" the Lorentz structure of the effective isovector interaction.
\end{abstract}

PACS numbers: 25.75.Dw, 24.10.Jv, 21.30.Fe, 21.65. $+\mathrm{f}$

Keywords: Asymmetric nuclear matter, isovector effective interactions, relativistic heavy ion collisions, particle production, subthreshold kaon production, $\left(K^{0} / K^{+}\right)$-ratio.

For at least two decades particle production has been suggested as a useful tool to constrain the poorly known high density behaviour of the nuclear equation of state $(E O S)$ 1, 2]. This information is crucial both to learn about the effective nuclear interaction and to get a deeper insight into many interesting astrophysical phenomena. In particular pion and (subthreshold) kaon productions have been extensively investigated both theoretically $2,[3,4,5,6,7,8]$ and experimentally $9,10,11$, leading to the conclusion of a soft behaviour of the $E o S$ at high densities, see the recent refs. [12, 13]. Kaons $\left(K^{0}, K^{+}\right)$appear as particularly sensitive probes since they are produced in the high density phase almost without subsequent reabsorption effects. At variance, antikaons $\left(\bar{K}^{0}, \bar{K}^{-}\right)$are strongly coupled to the hadronic medium through strangeness exchange reactions 12, 14, 15. In this paper we show that the isospin dependence of the $K^{0,+}$ production cross sections can be also used to probe the isovector part of the $E o S$ : we propose the $K^{0} / K^{+}$yield ratio as a good observable to constrain the high density behavior of the symmetry energy, $E_{\text {sym }}$, 16, 17.

In heavy ion collisions $(H I C \mathrm{~s})$ at intermediate energies (1-2 AGeV) a transient state of highly compressed and heated matter is created, with density up to 3-4 times the saturation value. Several dynamical observables have hence been proposed to characterize the density dependence of $E_{\text {sym }}$, such as charge ratios and collective isospin flows for highly energetic nucleons and pions 18, 19, 20, 21, 22, 23] and isospin transparency as expressed in transport ratios [24, 25, 26]. Controversial deductions arise from different relativistic and nonrelativistic models, see [17].
We show that, within a covariant description of nuclear dynamics, from the $K^{0} / K^{+}$ratios we can directly investigate the Lorentz structure, i.e. the scalar-vector decomposition, of the isovector sector of the effective in-medium hadron Lagrangian. Some promising indications have been recently obtained in nuclear matter calculations [27], here we present results for realistic open systems, i.e. for collisions of neutron-rich heavy ions in the energy range around the kaon production threshold (1.56 AGeV).

Using a transport model, derived within the Relativistic Mean Field approximation $(R M F)$ of QuantumHadro-Dynamics 28], we analyze pion and kaon production in central ${ }^{197} A u+{ }^{197} A u$ collisions in the $0.8-$ $1.8 \mathrm{AGeV}$ beam energy range, with different $R M F$ effective field choices for $E_{\text {sym }}$. We will compare results of three Lagrangians with constant nucleon-meson couplings ( $N L \ldots$ type, see $17,[20])$ and one with density dependent couplings ( $D D F$, see [20]), recently suggested for apparent better nucleonic properties of neutron stars [29]. In order to isolate the sensitivity to the isovector components we use models showing the same "soft" EoS for symmetric matter [17, 20. This is achieved via NonLinear $(N L \ldots)$ contributions of the isoscalar scalar $\sigma$ and vector $\omega$ mesons in the constant coupling cases, and via suitable density dependences in the $D D F$ model.

For the isovector part, in the simple $N L$ choice the mean field is not isospin dependent and the symmetry energy is just due to kinetic (Fermi) contributions. The $N L \rho$ model contains an isovector-vector effective field ( $\rho$ meson), which leads to a splitting of the vector selfenergies between protons and neutrons, and to a more repulsive force experienced by neutrons with respect to 
protons in neutron-rich matter [17]. In the $N L \rho \delta$ model also an isovector-scalar $\delta$ field is included, which gives an effective mass splitting between protons and neutrons 30, 31.

With both $\rho$ - and $\delta$-meson couplings, $f_{\rho, \delta}, E_{\text {sym }}$ can be written as 31 ]

$$
E_{\text {sym }}=\frac{1}{6} \frac{k_{F}^{2}}{E_{F}}+\frac{1}{2}\left[f_{\rho}-f_{\delta}\left(\frac{m^{*}}{E_{F}}\right)^{2}\right] \rho_{B} .
$$

leading to a partial cancellation of scalar and vector components, equivalent to that of the $\sigma$ and $\omega$ fields in the isoscalar sector. Since the $\delta$ field couples to the scalar density, $f_{\delta}$ is multiplied with a density dependent quenching factor in Eq. (11). It is then clear that the increase by nearly a factor of three in $f_{\rho}$ in the $N L \rho \delta$ model is necessary to compensate the attraction due to the scalar $\delta$ field, in order to get the same bulk asymmetry parameter $a_{4}=30.5 \mathrm{MeV}$ at saturation, 20, 31. Consequently the inclusion of a $\delta$ field leads to a stiffer symmetry energy at high baryon densities [31], and to larger vector self-energies for nucleons, as discussed in [19, 27]. In the $D D F$ model the $f_{\rho}$ is exponentially decreasing with density, resulting in a rather "soft" symmetry term at high density [20, 29].

We like to note the genuine relativistic nature of these mechanisms that will directly influence transport dynamics and particle production. A transport code with only relativistic kinematics combined to density dependent interactions will miss these effects.

In the energy range considered here the nucleonnucleon inelastic channels can be restricted to the excitation of the lowest mass resonance $\Delta(1232)$ and perturbative kaon $\left(K^{+, 0}\right)$ production through baryon-baryon collisions $B B \longrightarrow B Y K$, where $B$ stands for nucleons or resonances and $Y$ for hyperons $\left(\Lambda, \Sigma^{ \pm, 0}\right)$. Pions are produced via the decay of the $\Delta(1232)$ resonance and - after propagation and rescattering - can contribute to the kaon yield through collisions with baryons: $\pi B \longrightarrow Y K$. All these processes are treated within a relativistic hadronic transport model of Boltzmann-Uehling-Uhlenbeck type $(R B U U)$, i.e. including a hadron mean field propagation 32, 33. The latter point, which goes beyond the "collision cascade" picture, is essential for particle production yields since it directly affects the energy balance of the inelastic channels, as extensively analysed in the following. Further details can be found in ref. 27], where all the parametrizations used for the corresponding cross sections are also specified.

Due to the $K^{+, 0}$ long mean free path their in-medium widths can be expected to be small and an on-shell quasiparticle treatment appears suitable. Here, as in [27], we do not generally use potentials for kaons and propagate them as free particles. This simplification is justifiable, since our main aim here is to show how the Lorentz structure of the symmetry energy influences the $K^{0} / K^{+}$ratio,
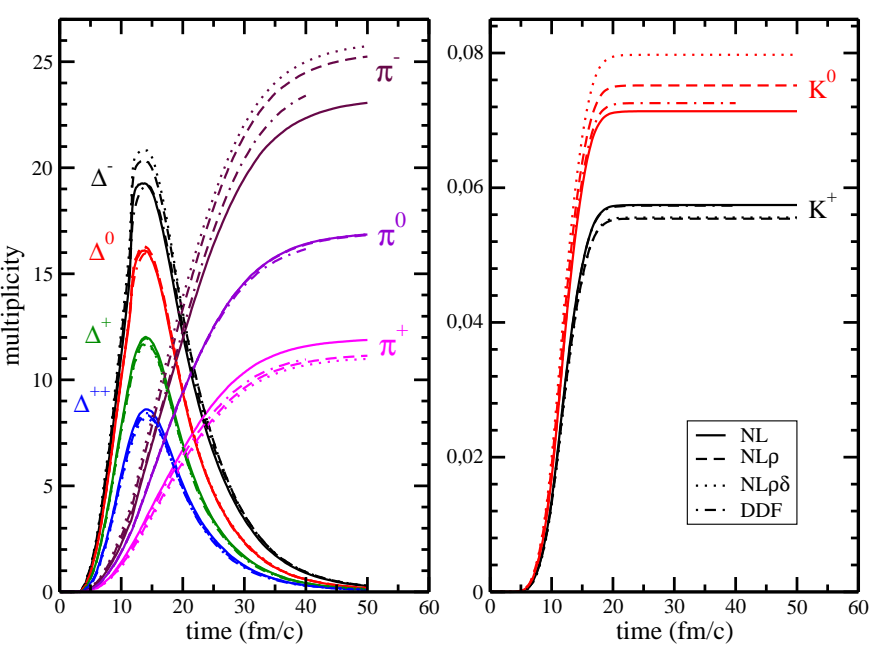

FIG. 1: Time evolution of the $\Delta^{ \pm, 0,++}$ resonances (left) and the pions $\pi^{ \pm, 0}$ (right) for a central ( $b=0$ fm impact parameter) $\mathrm{Au}+\mathrm{Au}$ collision at $1 \mathrm{AGeV}$ incident energy. Transport calculation using the $N L, N L \rho, N L \rho \delta$ and $D D F$ models for the iso-vector part of the nuclear EoS are shown.

which should not sensitively depend on the kaon-nucleon potential as this appears roughly the same (slightly repulsive) for both $K^{0}$ and $K^{+}[12$.

Fig. 1 reports the temporal evolution of $\Delta^{ \pm, 0,++}$ resonances and pions $\left(\pi^{ \pm, 0}\right)$ (left panel) and kaons $\left(K^{+, 0}\right)$ (right panel) for central $\mathrm{Au}+\mathrm{Au}$ collisions at $1 \mathrm{AGeV}$. Results are shown for all the tested models.

It is clear that, while the pion yield freezes out at times of the order of $50 \mathrm{fm} / \mathrm{c}$, i.e. at the final stage of the reaction (and at low densities), kaon production occurs within the very early stage of the reaction, and the yield saturates at around $20 \mathrm{fm} / \mathrm{c}$. Kaons are then suitable to probe the high density phase of nuclear matter. This is not the case for pions, which suffer from reabsorption and isospin exchange processes that modify both the absolute primordial yield and the $\pi^{-} / \pi^{+}$ratio. In fact from Fig. [1 we see that the results for different models are rather similar, and thus pion multiplicities depend only weakly on the isospin part of the nuclear mean field. However, a slight increase (decrease) in the $\pi^{-}\left(\pi^{+}\right)$multiplicity is observed when going from the $N L$ to the $N L \rho$ and then to the $N L \rho \delta$ model, i.e. increasing the vector contribution $f_{\rho}$ in the isovector channel. This trend is more pronounced for kaons, see Fig. 1 1right panel, due to the high density selection of the source and the proximity to the production threshold. The results for the DDF model, density dependent couplings with a large $f_{\rho}$ decrease at high density, are fully consistent. They are always closer to the $N L$ case (without isovector interactions) but the difference still seen for $\pi^{+,-}$is completely disappearing for $K^{0,+}$, selectively produced at high densities.

When isovector fields are included the symmetry potential energy in neutron-rich matter is repulsive for neu- 
trons and attractive for protons. In a $H I C$ this leads to a fast, pre-equilibrium, emission of neutrons towards more symmetric nuclear matter. Such a mean field mechanism, which has been extensively investigated, often referred to as isospin fractionation [16, 17], is responsible for a reduction of the neutron to proton ratio during the high density phase, with direct consequences on particle production in inelastic $N N$ collisions.

Threshold effects represent a more subtle question. The energy conservation in a hadron collision in general has to be formulated in terms of the canonical momenta, i.e. for a reaction $1+2 \rightarrow 3+4$ as

$$
s_{\text {in }}=\left(k_{1}^{\mu}+k_{2}^{\mu}\right)^{2}=\left(k_{3}^{\mu}+k_{4}^{\mu}\right)^{2}=s_{\text {out }}
$$

Since hadrons are propagating with effective (kinetic) momenta and masses, an equivalent equation should be formulated starting from the effective in-medium quantities $k^{* \mu}=k^{\mu}-\Sigma^{\mu}$ and $m^{*}=m+\Sigma_{s}$, where $\Sigma_{s}$ and $\Sigma^{\mu}$ are the scalar and vector self-energies, respectively, depending on the isovector channel structure. In particular, for the general $\sigma \omega \rho \delta$ case one obtains for the self-energies of protons and neutrons:

$$
\begin{aligned}
\Sigma_{s}(p, n) & =-f_{\sigma} \rho_{s} \pm f_{\delta} \rho_{s 3} \\
\Sigma^{\mu}(p, n) & =f_{\omega} j^{\mu} \mp f_{\rho} j_{3}^{\mu},
\end{aligned}
$$

(upper signs for neutrons), where $\rho_{s}=\rho_{s p}+\rho_{s n}, j^{\alpha}=$ $j_{p}^{\alpha}+j_{n}^{\alpha}, \rho_{s 3}=\rho_{s p}-\rho_{s n}, j_{3}^{\alpha}=j_{p}^{\alpha}-j_{n}^{\alpha}$ are the total and isospin scalar densities and currents and $f_{\sigma, \omega, \rho, \delta}$ are the coupling constants of the various mesonic fields, 34].

In reactions where nucleon resonances, especially the different isospin states of the $\Delta$ resonance, and hyperons enter, also their self energies are relevant for energy conservation. We specify them in the usual way according to the light quark content and with appropriate ClebschGordon coefficients [27].

In the most general case the isovector scalar and vector self-energies enter the new threshold condition for a given inelastic process [27]

$$
s_{i n} \geq\left(m_{3}^{*}+\Sigma_{3}^{0}+m_{4}^{*}+\Sigma_{4}^{0}\right)^{2}-\left(\boldsymbol{\Sigma}_{3}+\boldsymbol{\Sigma}_{4}\right)^{2} .
$$

The condition of energy conservation in inelastic hadron collisions will influence the particle production in two different ways. On one hand it will directly determine the thresholds and thus the multiplicities of a certain type of particles, in particular of the sub-threshold ones, as here for the kaons. Secondly it may favour or penalize reactions, because the self-energies in the final channel are more attractive or repulsive than in the initial one, and consequently the phase space in the final channel is larger or smaller.

Hence, we clearly see that the competition between scalar and vector isovector fields is responsible for the isospin fractionation and modifies particle production rates. In particular while the scalar and vector isovector fields tend to cancel in the symmetry term, see Eq.(11), they can have very different dynamical effects, as already noted in the flow analysis of ref. [19]. In fact mean field and threshold effects are acting in opposite directions on particle production and might compensate each other.

As an example, $n n$ collisions excite $\Delta^{-, 0}$ resonances which decay mainly to $\pi^{-}$. In a neutron-rich matter the mean field effect pushes out neutrons making the matter more symmetric and thus decreasing the $\pi^{-}$yield. The threshold effect on the other hand is increasing the rate of $\pi^{-}$'s due to the enhanced production of the $\Delta^{-}$resonances: now the $n n \rightarrow p \Delta^{-}$process is favored (with respect to $p p \rightarrow n \Delta^{++}$) since more effectively a neutron is converted into a proton.

We have to note that such interplay between the two mechanisms cannot be fully included in a non-relativistic dynamics, in particular in calculations where the baryon symmetry potential is treated classically [18, 21, 22, 23]. A typical example is the strength of the isovector-vector $\rho$-coupling which is linked to the symmetry energy but it is largely varying with the Lorentz structure of the isovector interaction. Actually aim of this paper is to show that the data could be used to directly probe the covariant structure of the effective interaction in the isovector channel at high baryon densities.

In the left panel of Fig. 11we see that $\Delta^{-}$(and $\pi^{-}$) production increases when going in the direction of larger $f_{\rho}$ couplings $N L \rightarrow D D F \rightarrow N L \rho \rightarrow N L \rho \delta$. As discussed before, the isospin fractionation lowers the $n / p$ ratio and thus decreases the $\Delta^{-}$(and $\pi^{-}$) yield, while the threshold mechanism increases it due to the larger $n n$ self-energies. In fact the latter effect appears to overcompensate the former. The opposite is true for the $\Delta^{+,++}\left(\right.$and $\pi^{+}$) production.

As seen in the right panel of Fig. 11 a similar argument holds for $K^{0}$ and $K^{+}$mesons, which mainly come from $n n$ (or $\pi^{-} n$ ) and $p p\left(\right.$ or $\pi^{+} p$ ) collisions, respectively, and thus exhibit the same trend as $\pi^{-}$and $\pi^{+}$. However, the isospin effect in this case is more pronounced because the changes in the self energies for the different models play a more crucial role close to the kaon production threshold.

Finally the beam energy dependence of the $\pi^{-} / \pi^{+}$ (left) and $K^{0} / K^{+}$(right) ratios is shown in Fig. 2] At each energy we see an increase of the yield ratios with the models $N L \rightarrow D D F \rightarrow N L \rho \rightarrow N L \rho \delta$. The effect is larger for the $K^{0} / K^{+}$compared to the $\pi^{-} / \pi^{+}$ratio. This is due to the subthreshold production and to the fact that the isospin effect enters twice in the two-steps production of kaons, see 35]. Between the two extreme $D D F$ and $N L \rho \delta$ isovector interaction models, the variations in the ratios are of the order of $14-16 \%$ for kaons, while they reduce to about $8-10 \%$ for pions. Interestingly the Iso-EoS effect for pions is increasing at lower energies, when approaching the production threshold.

We have to note that in a previous study of kaon production in excited nuclear matter the dependence of the 
$K^{0} / K^{+}$yield ratio on the effective isovector interaction appears much larger, about ten times more for a system with the $A u$ asymmetry (see Fig.8 of ref. 27] ). The point is that in the non-equilibrium case of a heavy ion collision the asymmetry of the source where kaons are produced is in fact reduced by the $n \rightarrow p$ "transformation", due to the favored $n n \rightarrow p \Delta^{-}$processes. This effect is almost absent at equilibrium due to the inverse transitions, see Fig.(3) of ref.[27]. Moreover in infinite nuclear matter even the fast neutron emission is not present.

Thus in an open system the signal appears weaker but still accessible experimentally, if it can be shown to be robust. In order to further stress the distinction between effects of the stiffness of the symmetry energy and the detailed Lorentz structure of the isovector part of the effective Lagrangian, we also show the results for the $K^{0} / K^{+}$with another parametrization of $E_{\text {sym }}$. This model, $N L D D \rho$, is a variant of $N L \rho$ with a density dependent $\rho$-coupling, built in such a way as to reproduce the same stiffer $E_{\text {sym }}\left(\rho_{B}\right)$ of the $N L \rho \delta$ model (see also ref. [19]). The results for the $\pi^{-} / \pi^{+}$and $K^{0} / K^{+}$ ratios are shown in Fig. 2 for $E_{\text {beam }}=1.0 \mathrm{AGeV}$ as triangles. We see that they are closer to the $N L \rho$ results (with a constant $f_{\rho}$ ) than to the ones of the $N L \rho \delta$ choice which has the same iso-stiffness. This nicely confirms that the differences observed going from the $N L \rho$ to the $N L \rho \delta$ parametrization are not due to the slightly increased stiffness of $E_{\text {sym }}\left(\rho_{B}\right)$, but more specifically to the competition between the attractive scalar $\delta$-field and the repulsive vector $\rho$-field in the isovector channel, which leads to the increase of the vector coupling (see the comments to the Eq.(1)). Hence the differences between the vector self-energies of neutrons and $\Delta^{-}$resonances vs. the proton and $\Delta^{++}$ones are greater in the $N L \rho \delta$ model than in other models without scalar isovector fields and the production cross sections are consequently affected. As a result, the subthreshold $K^{0}\left(K^{+}\right)$yield is sensitively enhanced (reduced) in the $N L \rho \delta$ case.

In the same Fig. 2] we also report results at $1.2 \mathrm{AGeV}$ for the ${ }^{132} S n+{ }^{124} S n$ reaction, induced by a radioactive beam, with an overall larger asymmetry (open symbols). The isospin effects are clearly enhanced. Finally we have performed some calculations including kaon potentials, derived from a chiral lagrangian as suggested in [12]. The $K^{0} / K^{+}$ratio appears not very sensitive to this in-medium modification of the kaon single particle energies. This is somewhat expected since we have a similar small repulsive contribution for both $K^{0,+}$ mesons.

From the present discussion we conclude that subthreshold kaon production could provide a promising tool to extract information on the isovector part of the nuclear interaction at high baryon density. We have seen that, at beam energies below and around the kinematical threshold, the $K^{0} / K^{+}$inclusive yield ratio is more sensitive to the Lorentz structure of $E_{\text {sym }}$ than the $\pi^{-} / \pi^{+}$.

We would like to stress the two most important re-

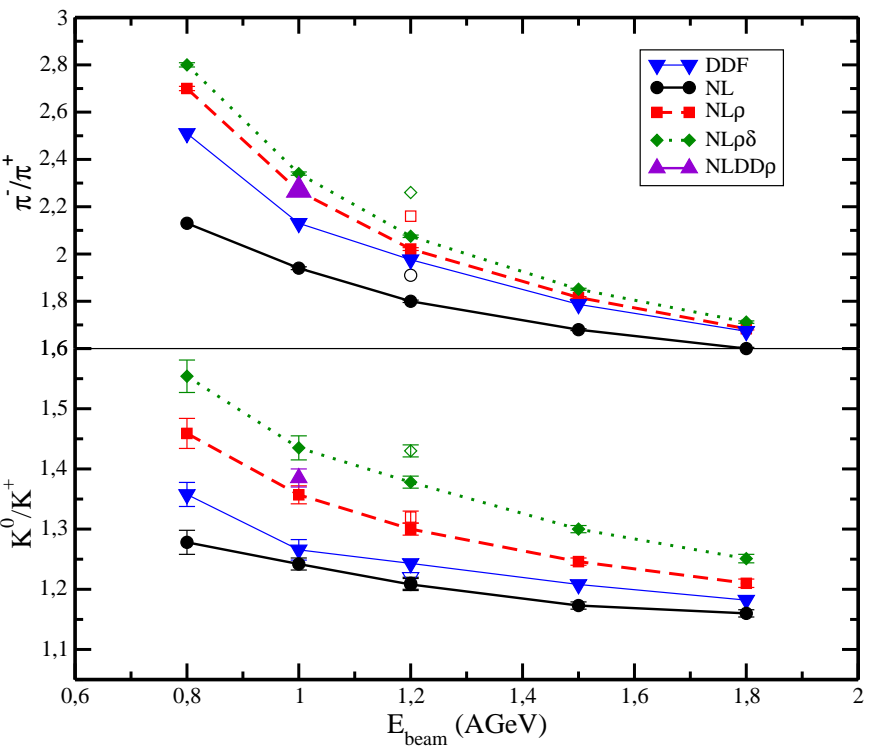

FIG. 2: $\pi^{-} / \pi^{+}$(upper) and $K^{+} / K^{0}$ (lower) ratios as a function of the incident energy for the same reaction and models as in Fig. 1 In addition we present, for $E_{\text {beam }}=1 \mathrm{AGeV}$, $N L \rho$ results with a density dependent $\rho$-coupling (triangles), see text. The open symbols at $1.2 \mathrm{AGeV}$ show the corresponding results for a ${ }^{132} S n+{ }^{124} S n$ collision, more neutron rich. Note the different scale for the $\pi^{-} / \pi^{+}$ratios.

sults of our study: i) We have shown that isospin effects are important not only at the mean-field level (isospin fractionation) but they also influence significantly particle production cross sections. As a matter of fact, we observe that, for the reactions studied here, the modifications induced in the inelastic vertices represent the dominant effects. ii) At relativistic energies, due to the Lorentz structure of the isovector nuclear interaction, the isotopic content of particle emission is not directly related to the symmetry energy value, but it can be rather considered as a measure of the strength of isovector vector channel.

We note that the isospin effects on the kaon inclusive yield ratios at the freeze-out appear not too strong, although experimentally accessible. It seems important to select more exclusive kaon observables, in particular with a trigger related to an early time $K$-production. A transverse momentum selection of pion yields, corresponding to a higher density source, should also be rather sensitive to isospin effects, in particular at lower energies, closer to the production threshold. A large asymmetry of the colliding matter is in any case of relevance. In this sense our work strongly supports the study of particle production at the new relativistic radioactive beam facilities. 


\section{Acknowledgments}

We would like to thank Christian Fuchs for providing us with the kaon package subroutines of the Tübingen group and for valuable discussions.

[1] R. Stock, Phys. Rep. 135, (1986) 259.

[2] J. Aichelin, C.M. Ko, Phys. Rev. Lett. 55, (1985) 2661.

[3] Ch. Hartnack et al., Nucl. Phys. A580 (1994) 643.

[4] T. Maruyama et al., Nucl. Phys. A573 (1994) 653.

[5] X.S. Fang et al., Nucl. Phys. A575 (1994) 766.

[6] G.Q. Li, C.M. Ko, Phys. Lett. B349 (1995) 405.

[7] C. Fuchs et al., Phys. Rev. C56 (1997) R606.

[8] C. Fuchs et al., Phys. Rev. Lett. 86, (2001) 1974.

[9] D. Miskowiec et al. (KaoS Collaboration), Phys. Rev. Lett. 72 (1994) 3650.

[10] F. Laue et al. (KaoS Collaboration), Phys. Rev. Lett. 82, 1640 (1999);

P. Senger, H. Ströbele, J. Phys. G 25 (1999) R59;

C. Sturm et al. (KaoS Collaboration), Phys. Rev. Lett. 86, 39 (2001).

[11] B. Hong et al., (FOPI collaboration), Phys. Rev. C71 (2005) 034902.

[12] C. Fuchs, Prog.Part.Nucl.Phys. 56, 1-103 (2006).

[13] C.Hartnack, H.Oeschler, J.Aichelin, Phys. Rev. Lett. 96 (2006) 012302.

[14] W. Cassing, L.Tolos, E.L. Bratkovskaya, A. Ramos, Nucl. Phys. A727, 59 (2003).

[15] H. Weber, E.L. Bratkovskaya, W. Cassing, W. Stöcker, Phys. Rev. C67, 014904 (2003).

[16] B.-A Li, W.U. Schroeder (Eds.), Isospin Physics in Heavy-Ion Collisions at Intermediate Energies, Nova Sci- ence, New York, 2001.

[17] V. Baran, M. Colonna, V. Greco, M. Di Toro, Phys. Rep. 410, 335 (2005).

[18] Bao-An Li, Nucl. Phys. A708, 365 (2002).

[19] V. Greco et al., Phys. Lett. B562, 215 (2003).

[20] T. Gaitanos et al., Nucl. Phys. A732, 24 (2004).

[21] B.-A. Li, G.-C. Yong, W. Zuo, Phys. Rev. C71 014608 (2005).

[22] Qingfeng Li et al., Phys. Rev. C72, 034613 (2005).

[23] Qingfeng Li et al., J. Phys. G32, 151 (2006).

[24] T. Gaitanos, M. Colonna, M. Di Toro, H.H. Wolter, Phys. Lett. B595, 209 (2004).

[25] Lie-Wen Chen, C.M. Ko, Bao-An, Li, Phys. Rev. C69, 054606 (2004).

[26] Qingfeng Li, Zhuxia Li, H.Stöcker, arXiv:nucl-th/0603050

[27] G. Ferini, M. Colonna, T. Gaitanos, M. Di Toro, Nucl. Phys. A762, 147 (2005).

[28] B. D. Serot, J. D. Walecka, Advances in Nuclear Physics, 16, 1, eds. J. W. Negele, E. Vogt, (Plenum, N.Y., 1986).

[29] T.Klähn et al. Constraints on the high-density nuclear equation of state ..., arXiv:nucl-th/0602038

[30] S. Kubis, M. Kutschera, Phys. Lett. B399, (1997) 191.

[31] B. Liu et al., Phys. Rev. C65, 045201 (2002).

[32] B. Blättel, V. Koch, U. Mosel, Rep. Prog. Phys. 56, 1 (1993).

[33] C. Fuchs, H.H. Wolter, Nucl. Phys. A589, 732 (1995).

[34] For the $N L$ models in Eq. (3) the scalar density should be replaced by $\sigma\left(\rho_{s}\right)$, solution of the non linear equation for the $\sigma$ field [17, 31].

[35] In the energy range explored here, the main contribution to the kaon yield comes from the pionic channels, in particular from $\pi N$ collisions, and from the $N \Delta$ channel, which together account for nearly $80 \%$ of the total yield, see [27]. 